\title{
CLOSED IDEALS IN GROUP ALGEBRAS
}

BY WALTER RUDIN ${ }^{1}$

Communicated October 26, 1959

Let $A(G)$ be the set of all Fourier transforms on the locally compact abelian group $G$, i.e., the set of all $f$ of the form

$$
f(x)=\int_{\Gamma}(x, \gamma) F(\gamma) d \gamma \quad\left(x \in G, F \in L^{1}(\Gamma)\right),
$$

where $\Gamma$ is the dual group of $G$ and $(x, \gamma)$ is the value of the character $\gamma$ at the point $x$. With the norm

$$
\|f\|=\int_{\Gamma}|F(\gamma)| d \gamma
$$

$A(G)$ is a commutative Banach algebra, and $G$ is its maximal ideal space.

If $I$ is a closed ideal in $A(G)$, let $Z(I)$ be the set of all $x \in G$ such that $f(x)=0$ for every $f \in I$. Malliavin $[3 ; 4 ; 5]$ has recently solved a problem of long standing by proving that in every nondiscrete $G$ there is a closed set $E$ such that $E=Z\left(I_{1}\right)=Z\left(I_{2}\right)$ for two distinct closed ideals $I_{1}$ and $I_{2}$ in $A(G)$. Combined with an older result of Helson [1] this implies that there are infinitely many closed ideals $I$ in $A(G)$ with $Z(I)=E$.

It is the purpose of this note to point out that Malliavin's construction for compact $G$ (he reduced the general case to this) yields an even more specific result:

Theorem. Suppose $G$ is an infinite compact abelian group. There is a real $f \in A(G)$ such that the closed ideals $I_{n}$ generated by the powers $f^{n}(n=1,2,3, \cdots)$ are all distinct.

We sketch the proof. If $g \in A(G)$ and $u$ is a real number, we define $a_{\gamma}(u)$ by

$$
e^{i u g(x)}=\sum_{\gamma \in \Gamma} a_{\gamma}(u) \cdot(x, \gamma) \quad(x \in G) .
$$

Malliavin [5] constructed a real $g \in A(G)$ for which

$$
\left|a_{\gamma}(u)\right|<\exp \left(-C|u|^{1 / 2}\right) \quad(\gamma \in \Gamma),
$$

where $C>0$ is independent of $\gamma$. (The exponent $1 / 2$ in (2) could be

\footnotetext{
1 Research Fellow of the Alfred P. Sloan Foundation.
} 
replaced by any $\lambda<1$, but not by 1 . Kahane's construction [2] should also be mentioned in this connection.) By (2),

$$
\sup _{\gamma \in \Gamma} \int_{-\infty}^{\infty}\left|a_{\gamma}(u) u^{n}\right| d u=M_{n}<\infty \quad(n=0,1,2, \cdots) .
$$

The mapping

$$
\phi \rightarrow \int_{G} \phi(g(x))(-x, \gamma) d x
$$

is, for each $\gamma$, a bounded linear functional in the space of all continuous functions $\phi$ on the range of $g$, and hence there are measures $\mu_{\gamma}$ on the line, with compact support, such that

$$
\int_{G} \phi(g(x))(-x, \gamma) d x=\int_{-\infty}^{\infty} \phi(t) d \mu_{\gamma}(t) .
$$

Taking $\phi(t)=e^{i u t}$, we see that $a_{\gamma}(u)$ is the Fourier-Stieltjes transform of $\mu_{\gamma}$, and (3) implies that $d \mu_{\gamma}(t)=m_{\gamma}(t) d t$, where each $m_{\gamma}$ is infinitely differentiable and

$$
\left|m_{\gamma}^{(n)}(t)\right| \leqq M_{n} \quad(\gamma \in \Gamma, t \text { real }) .
$$

Since $a_{0}(0)=1, m_{0} \neq 0$, and there is a real number $\alpha$ such that $m_{0}(\alpha) \neq 0$.

Put $f(x)=g(x)-\alpha$. By (6), the expressions

$$
T_{n} h=(-1)^{n} \sum_{\gamma \in \Gamma} H(\gamma) m_{\gamma}^{(n)}(\alpha) \quad(n=1,2,3, \cdots),
$$

where $h(x)=\sum H(\gamma)(x, \gamma)$, define bounded linear functionals on $A(G)$. The following two facts show that $T_{n}$ annihilates $I_{n+1}$ but not $I_{n}$, and hence establish the theorem:
(A) $T_{n} f^{n} \neq 0$.
(B) If $h(x)=\left(x, \gamma_{0}\right) f^{n+1}(x)$, for any $\gamma_{0} \in \Gamma$, then $T_{n} h=0$.
(A) and (B) are proved by evaluating (7) for all $h$ of the form

$$
h(x)=P(g(x))\left(x,-\gamma_{0}\right)
$$

where $P$ is a polynomial. Set

$$
c_{j, n}(\gamma)=\int_{-\infty}^{\infty} W_{j}^{(n)}(t) m_{\gamma}(t) d t
$$

where $\left\{W_{j}\right\}$ is a sequence of non-negative infinitely differentiable functions which vanish outside $(\alpha-1 / j, \alpha+1 / j)$, such that $\int_{-\infty}^{\infty} W_{j}(t) d t$ 
$=1$. Integrating (9) by parts $n$ times, we see that $\left|c_{j, n}(\gamma)\right| \leqq M_{n}$ and $\lim _{j} c_{j, n}(\gamma)=(-1)^{n} m_{\gamma}^{(n)}(\alpha)$. Hence (5) implies, if $h$ is of the form (8), that

$$
\begin{aligned}
T_{n} h & =\lim _{\boldsymbol{J}} \sum_{\gamma} H(\gamma) \int_{-\infty}^{\infty} W_{j}^{(n)}(t) m_{\gamma}(t) d t \\
& =\lim _{j} \sum_{\gamma} H(\gamma) \int_{G} W_{j}^{(n)}(g(x))(x, \gamma) d x \\
& =\lim _{\boldsymbol{J}} \int_{G} W_{j}^{(n)}(g(x)) P(g(x))\left(x,-\gamma_{0}\right) d x \\
& =\lim _{\mathcal{J}} \int_{-\infty}^{\infty} W_{j}^{(n)}(t) P(t) m_{\gamma_{0}}(t) d t \\
& =(-1)^{n} \lim _{j} \int_{-\infty}^{\infty} W_{j}(t)\left(\frac{d}{d t}\right)^{n}\left[P(t) m_{\gamma_{0}}(t)\right] d t \\
& =(-1)^{n}\left(\frac{d}{d t}\right)^{n}\left[P(t) m_{\gamma_{0}}(t)\right]_{t=\alpha} .
\end{aligned}
$$

Taking $h=(g-\alpha)^{n}$, it follows that $T_{n} f^{n}$ is the $n$th derivative of $(-1)^{n}(t-\alpha)^{n} m_{0}(t)$, evaluated at $t=\alpha$, and this is $(-1)^{n} n ! m_{0}(\alpha) \neq 0$. This proves $(\mathrm{A})$.

Taking $h(x)=\left(x, \gamma_{0}\right)(g(x)-\alpha)^{n+1}$, we see that $T_{n} h$ is the $n$th derivative of $(-1)^{n}(t-\alpha)^{n+1} m_{\gamma_{0}}(t)$, evaluated at $t=\alpha$, which is 0 . This proves (B).

\section{REFERENCES}

1. Henry Helson, On the ideal structure of group algebras, Ark. Mat. vol. 2 (1952) pp. 83-86.

2. J. P. Kahane, Sur un thêorème de Paul Malliavin, C. R. Acad. Sci. Paris vol. 248 (1959) pp. 2943-2944.

3. Paul Malliavin, Sur l'impossibilite de la synthèse spectrale dans une algèbre de fonctions presque périodigues, C. R. Acad. Sci. Paris vol. 248 (1959) pp. 1756-1759.

4. - Sur l'impossibilité de la synthèse spectrale sur la droite, C. R. Acad. Sci. Paris vol. 248 (1959) pp. 2155-2157.

5. - Impossibilitê de la synthèse spectrale sur des groupes abelliens non compacts, Publications Mathématiques de l'Institut des Hautes Etudes Scientifiques Paris, 1949, pp. 61-68.

UNIVERSITY OF WISCONSIN 